Original paper

\title{
Direct antiviral agents for chronic hepatitis $C$ virus infection improve health-related quality of life significantly in the long term
}

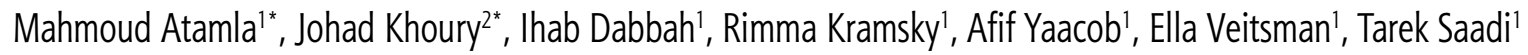 \\ 'Rambam Health Care Campus, Israel \\ 2Pulmonology Department, Lady Davis Carmel Medical Center, Israel \\ *These authors are equally contributed to this paper.
}

\begin{abstract}
Introduction: Direct antiviral agents (DAAs) are new drugs for the treatment of chronic hepatitis C virus (HCV) infection. These drugs are very effective and well tolerated. HCV can cause liver disease as well as extrahepatic manifestations, including a profound negative impact on health-related quality of life (HRQL).

Aim of the study: To evaluate HRQL in the long term (> 6 months after finishing treatment) after successful treatment with DAAs. To the best of our knowledge, this is the first study that evaluates quality of life in the long term after DAA treatment.

Material and methods: This is an observational study which included 100 patients treated with DAAs for chronic HCV infection between January 2015 and August 2018. Patients were assigned randomly. The average time after finishing treatment was 29.96 months. The Liver Disease Symptom Index (LDSI) 2.0 Questionnaire was used to evaluate quality of life before and after treatment.

Results: Seven of 9 parameters of the LDSI 2.0 Questionnaire showed significant improvement in the long term after successful treatment with DAAs. Two parameters (arthralgia and jaundice) did not improve significantly. Quality of life improved in both males and females similarly. Improvement did not correlate with the severity of liver fibrosis.

Conclusions: Treatment with DAAs improves HRQL significantly in the long term.

Key words: quality of life, HCV, hepatitis C virus, DAA, direct antiviral agent.
\end{abstract}

Address for correspondence:

Dr. Johad Khoury, Pulmonology Department, Lady Davis Carmel Medical Center, Michal St., 34362 Israel, e-mail: jihadfk@gmail.com

\section{Introduction}

Hepatitis $\mathrm{C}$ virus (HCV) infection is a worldwide health concern. Since chronic HCV infection may progress to advanced liver disease, cirrhosis and hepatocellular carcinoma (HCC), HCV-related mortality is estimated at about 500,000 deaths each year [1].

Hepatocellular carcinoma is highly correlated with liver fibrosis degree, and develops in $1-7 \%$ of cirrhotic patients $[2,3]$. HCV eradication can prevent the development of high stage liver fibrosis and decrease associated mortality $[4,5]$. HCV is also associated with extrahepatic manifestations, including cryoglob- ulinemia, porphyria cutanea tarda, diabetes mellitus, neuropsychiatric disorders [6], cognitive impairment, processing speed and concentration [7-9].

As a result of the effect of HCV on the liver as well as on other organ systems (extrahepatic manifestations of HCV), HCV has been widely reported to have a profound negative impact on health-related quality of life (HRQL), work productivity and other patient-reported outcomes [10].

In previous years, it has been shown that interferon IFN-based successful treatment, i.e. sustained viral response (SVR), correlated with better prognosis [11]. However, since the introduction of direct antiviral 
agents (DAAs), which are more tolerated and effective than INF, some concerns have been raised considering higher HCC incidence in these patients $[12,13]$, although the ANRS collaborative study group examined three large French prospective multicenter studies of HCV patients treated with DAAs and found no increased risk of HCC in patients treated with DAAs [14]. However, INF-based treatment had many side effects and was often discontinued because of these side effects. During treatment with INF, quality of life was seriously impaired [11].

The effect of treatment with DAAs on quality of life has been studied recently, with conflicting results. While Younossi et al. found that treatment with DAAs correlated with improvement in quality of life regardless of the fibrosis degree [10], Kawakubo et al. concluded that the quality of life did not change after similar treatment [15].

This study aims to discuss the long-term quality of life after DAA treatment as compared to baseline.

\section{Material and methods}

A study was conducted at Rambam Health Care Campus in Haifa, Israel, between January 2015 and August 2018. Every third patient was randomly assigned to the study. Background data, including HCV-related data, were gathered from the digital profiles both before treatment and six months after the treatment. The Model for End-stage Liver Disease (MELD) score was calculated for each patient. Glomerular filtration rate (GFR) was calculated by the modification of the diet in renal disease (MDRD) equation.

Quality of life was estimated by the Translating and Testing the Liver Disease Symptom Index II (LDSI 2.0) score (Appendix), which includes nine symptoms: itch, joint pain, right upper quadrant pain, sleepiness during the day, family worry, decreased appetite, depression, fear of complications, and jaundice. The severity of each symptom may vary from 0 (not at all) to 5 (very severe). This score is highly validated for severity of liver disease symptoms [16]. The study staff called every patient by telephone to verify the severity of symptoms before and after treatment.

The primary end point was the long-term effect of DAAs on the nine symptoms listed above. The secondary endpoints were the effect of DAAs on the average of LDSI 2.0 symptom scores in men and women, and in different fibrosis stages.

The study was approved by the local institutional review board. All the authors had access to the data and have reviewed and approved the final manuscript.

\section{Statistical analysis}

Processing and analysis of the statistical data was done with IBM SPSS statistical software, version 24. For continuous variables, the mean, median and standard deviations were calculated. The $t$-test and Mann-Whitney test were used to examine the differences between the continuous variables in the investigation groups. To investigate the categorical parameters, the $\chi^{2}$ test or Fisher's exact test was used. All the statistical tests were two-sided, with a $p$ value of $\leq 0.05$ considered as statistically significant. Data analysis was done after the end of data collection.

\section{Results}

One hundred patients were included in the study, which was conducted over a 30-month period. Background data are summarized in Table 1.

The vast majority $(84 \%)$ of the patients suffered from a high degree of fibrosis (F3/F4) according to

Table 1. Background data, before treatment was started

\begin{tabular}{lc}
\hline Parameter & Average \pm SD/number (\%) \\
\hline Age (years) & $58.1 \pm 13.3$ \\
\hline Male & $56(56 \%)$ \\
\hline HIV positive & $13(13 \%)$ \\
\hline HBV positive & $1(1 \%)$ \\
\hline Diabetes mellitus & $12(12 \%)$ \\
\hline Alcohol abuse & $5(5 \%)$ \\
\hline Post-transplant & $5(5 \%)$ \\
\hline Smoker & $28(28 \%)$ \\
\hline Albumin (mg/dl) & $4.0 \pm 0.49$ \\
\hline Bilirubin (g/dl) & $0.89 \pm 0.48$ \\
\hline INR & $1.03 \pm 0.11$ \\
\hline Platelets & $163.9 \pm 66.5$ \\
\hline Hemoglobin & $13.9 \pm 1.8$ \\
\hline White blood cells & $6.2 \pm 2.1$ \\
\hline Creatinine & $0.82 \pm 0.2$ \\
\hline GGT (U/l) & $131.9 \pm 90.8$ \\
\hline ALP (U/l) & $101.4 \pm 43.3$ \\
\hline ALT (U/l) & $98.4 \pm 86.6$ \\
\hline AST (U/l) & $91.2 \pm 81.4$ \\
\hline LDL (mg/dl) & $94.9 \pm 32.9$ \\
\hline HDL (mg/dl) & $49.2 \pm 12.9$ \\
\hline TRIG (mg/dl) & $119.1 \pm 54.2$ \\
\hline SD standadderan & \\
\hline
\end{tabular}

$S D$ - standard deviation, HIV - human immunodeficiency virus, $H B V$ - hepatitis B virus, INR - international normalized ratio, GGT - $\gamma$-glutamyltransferase, ALP - alkaline phosphatase, ALT - alanine aminotransferase, AST - aspartate aminotransferase, LDL - low-density lipoprotein, HDL - high-density lipoprotein, TRIG - triglycerides 


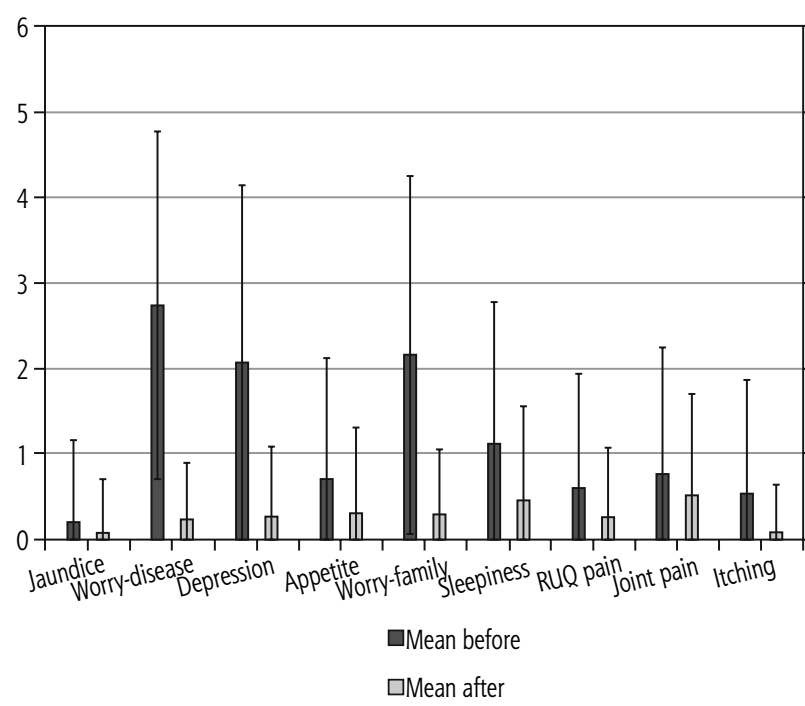

SD - standard deviation, RUQ - right upper quadrant, Sleepiness - day-time sleepiness, Worry-family - worry about family situation, Appetite - decreased appetite, Worry-disease worry about liver disease

Fig. 1. Mean and standard deviation of symptoms before and after treatment

the METAVIR score. The number (and percentage) of patients with $\mathrm{F} 0+\mathrm{F} 1, \mathrm{~F} 2, \mathrm{~F} 3$ and $\mathrm{F} 4$ fibrosis degree was $6(6 \%), 10(10 \%), 42(42 \%)$ and $42(42 \%)$ respectively. The most common genotype was G1B (84 (84\%) patients; the prevalence of the genotypes $1 \mathrm{~A}, 2,3$ and 4 was $4(4 \%), 1(1 \%), 10(10 \%)$ and $1(1 \%)$ respectively. Three patients suffered from ascites (3\%), 16 from esophageal varices (16\%) and one from hepatic encephalopathy (1\%).

Sixty patients (60\%) were treated with paritaprevir, ombitasvir, ritonavir \pm dasabuvir, one (1\%) with simeprevir, sofosbuvir, 12 (12\%) with each of ledipasvir, sofosbuvir, and daclatasvir, sofosbuvir, nine (9\%) with elbasvir, grazoprevir and six (6\%) with sofosbuvir, velpatasvir. Nine patients (9\%) received 24-week therapy, and the rest were treated for 12 weeks. None of the patients had previous major mental illness, while 13 were previous intravenous drug users.

Regarding quality of life, significant improvement was seen in 7/9 parameters, including itching, pain in the right upper abdomen, sleepiness during the day, worry about family situation, decreased appetite, de-

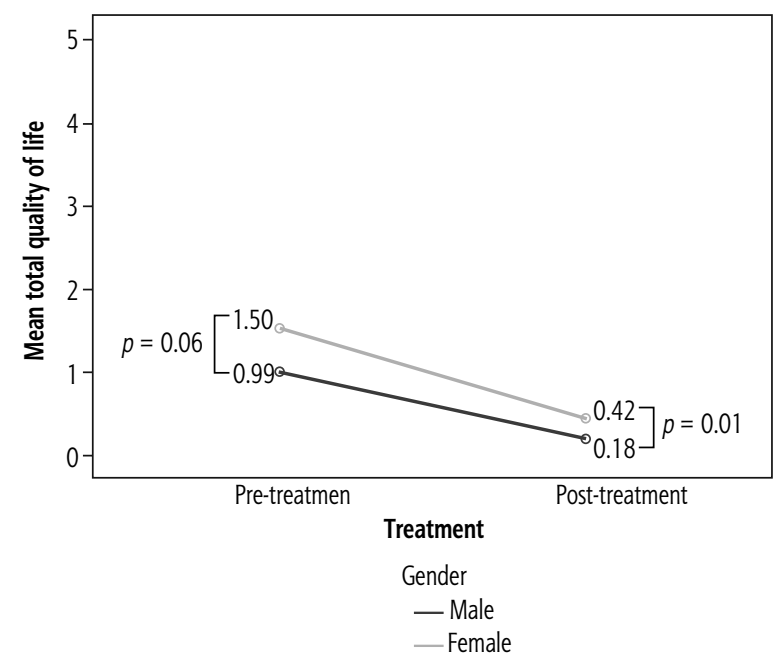

Fig. 2. Mean quality of life in males and females before and after treatment

pression and worry about liver disease complication. However, joint pain and jaundice did not change significantly after treatment (Fig. 1).

The mean value of the nine questions, which may be used as a summary of quality of life, was $1.22 \pm 0.95$ before treatment, median 1.1 (0-3.78); after treatment it was reduced to a mean of $0.29 \pm 0.47$, median 0.11 (0-2.78), $p=0.001$.

When subdividing the cohort by gender, men had a better quality of life than women, both before and after treatment. Quality of life improved after treatment in both gender groups (Fig. 2).

While assessing the quality of life in different fibrosis stages, life improved significantly after treatment in all fibrosis stages (Table 2, Fig. 3). All the fibrosis groups had a similar post-treatment quality of life.

An inverse relationship was found between age and quality of life, which was statistically significant $(p=0.012)$ but weak $(R=0.252)$ (Fig. 4$)$.

Some laboratory data changed after treatment. Alanine aminotransferase (ALT) and aspartate aminotransferase (AST) declined from 98 and $91 \mathrm{U} / \mathrm{l}$, respectively, to 35 and $29 \mathrm{U} / 1, p<0.001$. Moreover, bilirubin and hemoglobin declined from $0.91 \mathrm{mg} / \mathrm{dl}$ and $13.8 \mathrm{~g} / \mathrm{dl}$ to $0.75 \mathrm{mg} / \mathrm{dl}$ and $13.28 \mathrm{~g} / \mathrm{dl}$, while white blood cells

Table 2. Mean LDSI II score in different fibrosis stages

\begin{tabular}{cccc}
\hline Fibrosis degree & Number of patients & Pre-treatment mean LDSI II score \pm SD & Post-treatment mean LDSI II score \pm SD \\
\hline F0 $+F 1$ & 6 & $1.63 \pm 0.43$ & $0.48 \pm 0.54$ \\
\hline F2 & 10 & $0.90 \pm 0.68$ & $0.23 \pm 0.56$ \\
\hline F3 & 42 & $1.12 \pm 0.9$ & $0.24 \pm 0.39$ \\
\hline F4 & 42 & $1.33 \pm 1.08$ & $0.32 \pm 0.53$ \\
\hline Total & 100 & $1.22 \pm 0.95$ & $0.29 \pm 0.47$ \\
\hline
\end{tabular}




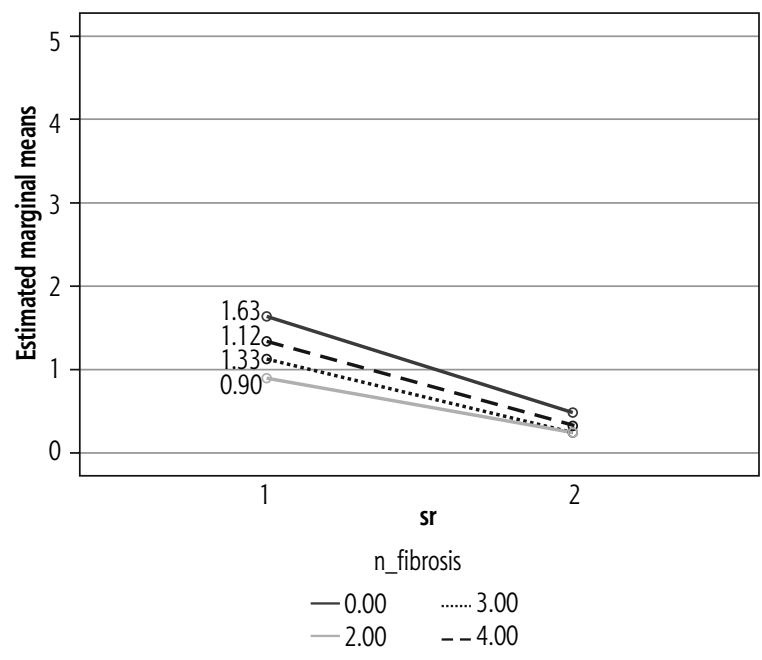

Fig. 3. Mean quality of life in different fibrosis stages

rose from 6 to $6.79 \times 10^{3}$ cells $/ \mathrm{dl}$. On the other hand, GFR declined from $95.7 \mathrm{ml} / \mathrm{min}$ before treatment, to $91.4 \mathrm{ml} / \mathrm{min}$ after treatment. All these changes were statistically significant, but clinically insignificant. Platelets did not change significantly.

\section{Discussion}

There has been huge progress in the treatment of HCV in recent years. Treatment with DAAs is very effective and tolerated with minimal adverse events. In the past, the treatment of $\mathrm{HCV}$ included pegylated-interferon (PEG-IFN) and ribavirin (RBV). This treatment was very difficult for patients and caused multiple adverse events. During treatment, quality of life was impaired. On the other hand, after reaching SVR, the quality of life of these patients was improved compared to their status before reaching SVR [17-23]. To date, there are no studies which examined the effect of DAAs on the long-term quality of life of these patients.

Our study observed the long-term influence of DAA treatment in 100 patients who had finished treatment with DAAs at least 6 months before enrollment, and some were three years after completion of treatment. Mean time between the start of the study and the end of the antiviral therapy was over 29 months.

This study showed a significant improvement in the long-term quality of life after treatment with DAAs. In addition, statistically significant improvements were seen in some of the laboratory tests, as expected. We also found that the improvement in quality of life was not related to gender or fibrosis stage.

A number of studies have evaluated quality of life after treatment with DAAs in the short term. To the best

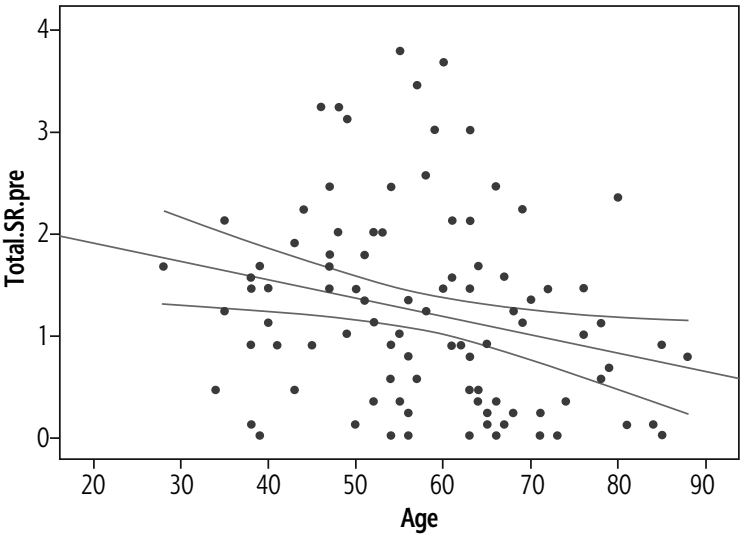

Fig. 4. Correlation between age and pre-treatment quality of life

of our knowledge, our study is the first to evaluate quality of life in the long term.

A previous study examined HRQL in 28 patients with chronic hepatitis $\mathrm{C}$ who received daclatasvir and asunaprevir for 24 weeks. The study showed that this combination improves quality of life in the short term. In addition, the nutritional status and liver fibrosis were improved in these patients and, therefore, the authors concluded that DAAs can induce long-term improvements in quality of life. Quality of life was assessed at three time points: before starting treatment, and at 12 and 24 weeks after completion of treatment [24].

Another cross-sectional study involved 147 Brazilian patients and compared quality of life with treatment with PEG-IFN and RBV vs. treatment with DAAs. Most (69\%) received PEG-IFN and RBV dual therapy and the remaining patients received DAAs. During treatment, quality of life was impaired by IFN-based therapies due to a significant number of side effects and also due to injections of IFN. On the other hand, quality of life did not change in patients who received DAAs [25].

The effect of IFN-free treatments on quality of life in patients with chronic hepatitis $\mathrm{C}$ was examined in a study of 686 patients who received PEG-IFN, sofosbuvir (SOF) and RBV for 12 weeks $(n=155)$ or SOF + RBV for $12-24$ weeks $(n=531)$. Eastern Asian HCV patients had significant impairment of HRQL during IFN treatment. Conversely, IFN removal from the treatment protocols significantly improved their quality of life [26].

In our study there was no significant improvement in joint pain in the patients after successful treatment. This finding is surprising because there is a significant association between joint pain and arthritis and chronic HCV infection. The sample size may not have been sufficient to estimate this point. Moreover, the severity of this symptom at baseline was low in our cohort. 
Another finding in our study was deterioration of renal function after SVR. We expected an opposite result since there is a correlation between infection with $\mathrm{HCV}$ and kidney diseases. The patients in our study had normal renal function before beginning treatment with DAAs, and this may be the reason why we did not see improvement of renal function after SVR. This issue needs to be assessed in a larger group of patients, especially patients with renal impairment before treatment.

An improvement in quality of life in our cohort was seen in both genders. It is important to note that the quality of life of women with HCV was lower than that of men before treatment. Even after treatment, the improvement in quality of life in women did not reach a similar level to men. In addition, improvement in quality of life was found to be significant in patients with mild liver fibrosis as well as in patients with advanced fibrosis, including cirrhotic patients.

Our study has several limitations: this is a singlecenter, retrospective study. Patients were treated with different antiviral protocols. Moreover, the questionnaires were undertaken at a point of time but not on several occasions, so that the results might be influenced by acute events.

In conclusion, DAAs are very effective and well-tolerated drugs for treating chronic HCV patients and they significantly improved patients' quality of life in the long term.

\section{Disclosure}

The authors declare no conflict of interest.

\section{References}

1. Saadi T, Khoury J. Is there a relationship between treatment with direct antiviral agents for HCV infection and the development of malignancies? J Clin Gastroenterol 2018; 52: 353-359.

2. Yang JD, Roberts LR. Hepatocellular carcinoma: a global view. Nat Rev Gastroenterol Hepatol 2010; 7: 448-458.

3. Yoshida H, Shiratori Y, Moriyama M, et al. Interferon therapy reduces the risk for hepatocellular carcinoma: national surveillance program of cirrhotic and noncirrhotic patients with chronic hepatitis C in Japan. IHIT Study Group. Inhibition of Hepatocarcinogenesis by Interferon Therapy. Ann Intern Med 1999; 131: 174-181.

4. Cheng EY, Saab S, Holt CD, et al. Paritaprevir/ritonavir/ombitasvir and dasabuvir for the treatment of chronic hepatitis $\mathrm{C}$ virus infection. Expert Opin Pharmacother 2015; 16: 2835-2848.

5. Younossi ZM, Singer ME, Mir HM, et al. Impact of interferon free regimens on clinical and cost outcomes for chronic hepatitis $\mathrm{C}$ genotype 1 patients. J Hepatol 2014; 60: 530-537.

6. Younossi ZM, Kanwal F, Saab S, et al. The impact of hepatitis C burden: an evidence-based approach. Aliment Pharmacol Ther 2014; 39: 518-531.

7. Morais-de-Jesus M, Daltro-Oliveira R, Pettersen KM, et al. Hepatitis C virus infection as a traumatic experience. PLoS One 2014; 9: e110529.
8. Quarantini LC, Miranda-Scippa A, Rocha M, Bressan RA. Neuropsychological function in patients with chronic hepatitis C. Liver Int 2008; 28: 893-894.

9. Batista-Neves SC, Quarantini LC, de Almeida AG, et al. High frequency of unrecognized mental disorders in HCV-infected patients. Gen Hosp Psychiatry 2008; 30: 80-82.

10. Younossi ZM, Stepanova M, Afdhal N, et al. Improvement of health-related quality of life and work productivity in chronic hepatitis $\mathrm{C}$ patients with early and advanced fibrosis treated with ledipasvir and sofosbuvir. J Hepatol 2015; 63: 337-345.

11. Shirabe K, Sugimachi K, Harada N, et al. Favorable prognosis in patients with sustained virological response to antiviral therapy, including interferon, for chronic hepatitis $\mathrm{C}$ before hepatic resection for hepatocellular carcinoma. Anticancer Res 2015; 35: 6963-6969.

12. Conti F, Buonfiglioli F, Scuteri A, et al. Early occurrence and recurrence of hepatocellular carcinoma in HCV-related cirrhosis treated with direct-acting antivirals. J Hepatol 2016; 65: 727-733.

13. Reig M, Marino Z, Perello C, et al. Unexpected high rate of early tumor recurrence in patients with HCV-related HCC undergoing interferon-free therapy. J Hepatol 2016; 65: 719-726.

14. ANRS collaborative study group on hepatocellular carcinoma (ANRS CO22 HEPATHER, CO12 CirVir and CO23 CUPILT cohorts). Lack of evidence of an effect of direct-acting antivirals on the recurrence of hepatocellular carcinoma: Data from three ANRS cohorts. J Hepatol 2016; 65: 734-740.

15. Kawakubo M, Eguchi Y, Okada M, et al. Chronic hepatitis C treatment with daclatasvir plus asunaprevir does not lead to a decreased quality of life. Intern Med 2018; 57: 1959-1966.

16. Van der Plas SM, Hansen BE, de Boer JB, et al. The Liver Disease Symptom Index 2.0; validation of a disease-specific questionnaire. Qual Life Res 2004; 13: 1469-1481.

17. Ware JE, Bungay K, Gandek B, Bayliss M. Assessment of the health related quality of life (HQL) of patients with chronic hepatitis C (CHC). Gastroenterology 1994; 106: A393.

18. Davis GL, Balart LA, Schiff ER, et al. Assessing health-related quality of life in chronic hepatitis $\mathrm{C}$ using the sickness impact profile. Clin Ther 1994; 16: 334-343.

19. Carithers RL, Sugano D, Bayliss M. Health assessment for chronic HCV infection. Dig Dis Sci 1996; 41 (Suppl): 75S-80S.

20. Bayliss M, Gandek B, Bungay KM, et al. A questionnaire to assess the generic and disease-specific health outcomes of patients with chronic hepatitis C. Qual Life Res 1998; 7: 39-55.

21. Foster GR, Goldin RD, Thomas HC. Chronic hepatitis C virus infection causes a significant reduction in quality of life in the absence of cirrhosis. Hepatology 1998; 27: 209-212.

22. Hunt C, Dominitz J, Bute BP, et al. Effect of interferon treatment of chronic hepatitis C on health-related quality of life. Dig Dis Sci 1997; 42: 2482-2486.

23. Bonkovsky HL, Woolley JM. Reduction of health-related quality of life in chronic hepatitis $\mathrm{C}$ and improvement with interferon therapy. Hepatology 1999; 29: 264-270.

24. Ohashi K, Ishikawa T, Suzuki M, et al. Health-related quality of life on the clinical course of patients with chronic hepatitis $\mathrm{C}$ receiving daclatasvir/asunaprevir therapy: a prospective observational study comparing younger $(<70)$ and elderly $(\geq 70)$ patients. Exp Ther Med 2018; 15: 970-976.

25. Perlin CM, Ferreira VL, Borba HHL, et al. Quality of life in Brazilian patients with treated or untreated chronic hepatitis C. Rev Inst Med Trop Sao Paulo 2017; 59: e81.

26. Younossi ZM, Stepanova M, Henry L, et al. The effect of interferon-free regimens on health-related quality of life in East Asian patients with chronic hepatitis C. Liver Int 2018; 38: 1179-1187. 


\section{Appendix}

To what extent in the past week:

Not at all

Has joint pain hampered you in your work or daily activities?

To what extent in the past week:

Not at all $ロ$ aロ

Did you have pain in the right upper belly?

To what extent in the past week:

Not at all $\square \square \square \square$ To a high extent

Has pain in the right upper belly hampered you in your work or daily activities?

To what extent in the past week: Were you sleepy during the day?

Not at all $\square \square \square \square$ To a high extent

To what extent in the past week:

Not at all $\square \square \square \square$ To a high extent

Has sleepiness hampered you in your work or daily activities?

To what extent in the past week:

Did you worry about the impact your liver disease may have on your home situation?

Not at all $ロ \square ロ \square T o$ a high extent

To what extent in the past week:

Not at all $ロ \square ロ \square T 0$ a high extent

Did your worrying about the impact your liver disease may have on your home family situation,

hamper you in your work or daily activities?

To what extent in the past week:

Did you have a decreased appetite?

To what extent in the past week:

Not at all $ロ$ ロロTo a high extent

Did decreased appetite hamper you?

To what extent in the past week:

Did you feel depressed due to your disease?

Not at all $ロ$ ロロ To a high extent

To what extent in the past week:

Not at all $\square \square \square \square T o$ a high extent

Did depression due to your disease hamper you in your work, daily activities and or social contacts?

To what extent in the past week:

Not at all $\square \square \square \square T 0$ a high extent

Were you afraid that possible liver disease complications would develop?

To what extent in the past week:

Not at all $\square \square \square \square T o$ a high extent

Did you skin turn yellow?

To what extent in the past week:

Not at all $\square \square \square \square T 0$ a high extent

Did yellowness of your skin hamper you in your work, daily activities and/or social contacts? 